\title{
Claudin-derived peptides are internalized via specific endocytosis pathways
}

\author{
Denise Zwanziger, ${ }^{1,}{ }^{*}$ Christian Staat, ${ }^{1,}{ }^{*}$ Anuska V. Andjelkovic, ${ }^{2}$ and Ingolf E. Blasig ${ }^{1}$ \\ ${ }^{1}$ Leibniz Institut für Molekulare Pharmakologie, Berlin-Buch, Germany. ${ }^{2}$ Department of Pathology, University of Michigan, \\ Medical School, Ann Arbor, Michigan
}

Address for correspondence: Ingolf E. Blasig, Leibniz Institut für Molekulare Pharmakologie, Robert-Rössle-Str. 10, 13125, Berlin-Buch, Germany. iblasig@fmp-berlin.de

\begin{abstract}
Claudin proteins are involved in the paracellular tightening of epithelia and endothelia. Their internalization, which can be modulated by extracellular stimuli, for example, proinflammatory cytokines, is a prerequisite for the regulation of the paracellular barrier to allow, for instance, cell migration or drug delivery. The internalization of peptide sequences of claudins is completely unknown. Here, we studied the internalization of two peptides, TAMRAclaudin-1 and TAMRA-claudin-5, derivatives of the extracellular loop of claudin-1 and -5 , respectively, in either epithelial or endothelial cells. The cellular uptake of the claudin-1 peptide follows the clathrin-mediated endocytosis as indicated by inhibitors and respective tracers for colocalization. In addition, macropinocytosis and caveolaemediated endocytosis of the peptide was observed. In contrast, the claudin-5 peptide is mainly internalized via the caveolae-mediated endocytosis evidenced by the colocalization with respective tracers and vesicle markers, whereas the nonselective macropinocytosis seems to be involved in a less effective manner. In conclusion, the assumption is supported that claudin peptides can be internalized by specific and nonspecific pathways.
\end{abstract}

Keywords: internalization; endocytosis; tight junction; claudin peptides; clathrin mediated; caveolae mediated

\section{Introduction}

Transmembrane tight junction (TJ) proteins seal the paracellular cleft between epithelial and endothelial cells. ${ }^{1-3}$ Moreover, TJ proteins regulate the paracellular flux of fluids and solutes. ${ }^{1-3}$ However, little is known about their intracellular function. Members of these proteins are claudin proteins, occludinlike proteins, and junctional adhesion molecules (JAM). Consequently, proteins are located at cellcell contact membranes of opposing cells and form a paracellular barrier. In contrast to the distinct organization, there is a continuous flux of the proteins by endocytosis followed by either recycling and incorporation back to the membrane or degradation and de novo synthesis. ${ }^{4}$ Endocytosis of the TJ proteins can influence the paracellular barrier integrity either due to one subtype, which is internalized,

*These authors contributed equally. or due to different subtypes, such as claudins and occludin. ${ }^{4,5}$

The intracellular uptake of $\mathrm{TJ}$ proteins can take place by three different endocytosis pathways, caveolae-mediated, clathrin-mediated, or more unspecifically, by macropinocytosis. ${ }^{6-8}$ The endocytosis pathways differ between TJ proteins and can be influenced by an extracellular stimulus, for example proinflammatory cytokines. ${ }^{9}$ The tumor necrosis factor- $\alpha$ or interferon- $\gamma$ induce internalization of claudin-1, zonula occludens protein 1 (ZO-1) or JAM-A by clathrin-mediated endocytosis and by macropinocytosis. ${ }^{10,11}$ By using a nonphysiological stimulus, like $\mathrm{Ca}^{2+}$ depletion, the clathrin-mediated endocytosis of several TJ proteins, for instance occludin and JAM-1, is initiated. ${ }^{12}$ In contrast, caveolae-mediated endocytosis and macropinocytosis have been observed for occludin and claudin-5 by the Escherichia coli toxin cytotoxic necrotizing factor-1 or the chemokine ligand $2 .^{4,13}$ However, nothing is known about the 
internalization pathway of peptides designed from claudin segments.

The 27 members of the claudin family are the major component of TJ strands. ${ }^{14}$ Their expression pattern varies between different tissues and these proteins are regulating the tissue-, charge-, and size-selectivity. ${ }^{15,16}$ In the blood-brain barrier, claudin-5 is the significant claudin subtype, ${ }^{17}$ whereas claudin-1 is essential for the tightness of the epidermal barrier. ${ }^{18}$ Claudins contain four transmembrane helices, an intracellular $\mathrm{N}$ - and Cterminus as well as one intracellular loop and two extracellular loops (ECL). The first ECL is with $\sim 50$ amino acids much longer than the second one with $\sim 10-20$ amino acids. They can interact with each other between the cell membranes of opposing cells (trans-interaction). ${ }^{19}$ Claudins are able to interact in a homophilic (same claudin subtype) or heterophilic (different claudin subtypes) manner. However, the complete mechanism of interaction is still unclear but both ECL seem to be involved. ${ }^{19-21}$

In this study, we investigated two fluorescently labeled peptides derived from the ECL of claudin-1, the 5,6-carboxytetramethylrhodamine (TAMRA)claudin-1 peptide, and derived from the ECL of claudin-5, the TAMRA-claudin-5 peptide, with respect to their internalization behavior. As claudin-1 is a key claudin for the epithelium and claudin5 for the endothelium epithelial and endothelial cell lines were used. Both peptides are internalized within few minutes and appear vesicle-like distributed. The claudin-1-derived peptide enters the cell via the clathrin-mediated endocytosis and macropinocytosis as well as to lower amounts by the caveolae-mediated endocytosis. In contrast, a caveolae-mediated endocytosis but no clathrinmediated endocytosis, and low macropinocytosis could be observed for the uptake of the claudin-5-derived peptide. Furthermore, our results predominantly agree with data regarding the internalization of the full-length proteins of claudin1 and claudin-5.

\section{Methods}

\section{Peptide synthesis}

Peptides were generated automatically (ACTIVOP11, Activotec.com, Cambridge, UK) by solid-phase peptide synthesis using $\mathrm{Fmoc} /{ }^{t} \mathrm{Bu}$ chemistry. Synthesis followed in a batch-wise mode at a distinct temperature with coupling times of $10 \mathrm{~min}$ at
$70{ }^{\circ} \mathrm{C}$ and the Fmoc-removal for seven minutes at $70{ }^{\circ} \mathrm{C}$ on Peg-resins (SRAM, $0.2 \mathrm{mmol} / \mathrm{g}$, Rapp-Polymere [Sigma-Aldrich, Germany]). TAMRA was introduced N-terminally after Fmocremoval to visualize the cellular pathway. Firstly, TAMRA (5 equiv. per Peg-resin, $0.1 \mathrm{mmol}$ ) was activated by 2 -(6-chloro-1H-benzotriazole-1-yl)1,1,3,3-tetramethylaminium hexafluoro-phosphate $(0.1 \mathrm{mmol})$ and $N, N$-diisopropylethylamine $(0.2$ $\mathrm{mmol}$ ) and then coupled for $40 \mathrm{~min}$ in dimethylsulfoxide with the respective resin. The cleavage from the resin occurred with trifluoroacetic acid (TFA) $/ \mathrm{H}_{2} \mathrm{O}(9 / 1)$ for three hours at room temperature. Then, peptides were precipitated with cold diethyl ether and purified by preparative reversed phase high-performance liquid chromatography (C-18 column) (Dionex, Sunnyvale, CA) with acetonitrile gradients in aqueous $0.1 \%$ TFA. Purified peptides were quantified and characterized by liquid chromatography (ACQUITY UPLC system, C18 column) electrospray time-of-flight mass spectrometry (LCT Premier, Waters, Germany), which showed a purity $>95 \%(220 \mathrm{~nm})$ and gave the expected masses. For in vitro experiments, TAMRA-labeled peptides were dissolved in dimethyl sulfoxide (final concentration of $0.1 \%$ ), and afterward added to the corresponding medium containing pluronic ${ }^{\circledR}$ F-127 $(0.012 \%)$ (Sigma-Aldrich, Germany).

\section{Cell culture}

Human embryonic kidney (HEK-293) cells ${ }^{22}$ stably transfected with claudin-1-yellow fluorescence protein (YFP) and immortalized mouse brain endothelial cells (bEnd.3) $)^{23}$ were grown at $37{ }^{\circ} \mathrm{C}$ and $10 \% \mathrm{CO}_{2}$. The transfection was thought to visualize colocalization between the claudin and the peptide tested. HEK-293 claudin-1-YFP cells were cultured in Dulbecco's modified Eagle's medium (DMEM) containing $10 \%$ fetal calf serum, 100 units $/ \mathrm{mL}$ penicillin, $100 \mu \mathrm{g} / \mathrm{mL}$ streptomycin, and 4 $\mathrm{mM}$ L-analyl-L-glutamine. bEnd. 3 cells and bEnd. 3 caveolin-1-green fluorescent protein (GFP) cells were cultured in DMEM containing 10\% fetal calf serum, $1 \times$ nonessential amino acids, and $2 \mathrm{mM}$ L-glutamine.

\section{Inhibitor study}

HEK-293 claudin-1-YFP cells were transferred into $35 \mathrm{~mm}$ tissue culture dishes containing poly-L-lysine-coated cover-slips and grown to 
subconfluence. Cells were pretreated with the respective inhibitor: chlorpromazine, ${ }^{24}$ as inhibitor of the clathrin-mediated endocytosis with $15 \mu \mathrm{M}$ for $30 \mathrm{~min}$, wortmannin ${ }^{25}$ with $250 \mathrm{nM}$ for $60 \mathrm{~min}$ or LY294002 with $100 \mu \mathrm{M}$ for $60 \mathrm{~min}$ as macropinocytosis inhibitors, and filipin ${ }^{26}$ as inhibitor of the caveolae-mediated endocytosis with $1 \mu \mathrm{g} / \mathrm{mL}$ for 30 minutes. Afterward, cells were treated with the TAMRA-claudin-1 peptide ( $40 \mu \mathrm{M})$ and the respective inhibitor for 35 minutes. Nuclei were stained with $5 \mu \mathrm{M}$ Hoechst 33342 (Life Technologies, Germany). Then, cells were washed three times with Hank's buffered salt solution and analyzed by quantification of the intracellular amount of peptide with the laser scanning microscope 510 Meta confocal microscope (Zeiss, Germany).

\section{Tracer study}

Colocalization studies of TAMRA-claudin-1: HEK293-claudin-1-YFP cells were seeded as described above. The clathrin internalization tracer Alexa680transferrin $(25 \mu \mathrm{g} / \mathrm{mL})$ (Invitrogen, Carlsbad, CA) and $40 \mu \mathrm{M}$ peptide were dissolved into medium and added to the apical side of HEK-293 claudin-1-YFP cells. After $25 \mathrm{~min}$ at $37^{\circ} \mathrm{C}$, samples were analyzed by confocal microscopy. Colocalization studies of TAMRA-claudin-5: The macropinocytosis marker, lysine-fixable Alexa Fluor 488-dextran ${ }^{12}(1 \mathrm{mg} / \mathrm{mL})$, the caveolae internalization marker, bodipy- $\mathrm{FL}^{4}$ $(5 \mu \mathrm{M})$, and the clathrin internalization marker, FITC-transferrin ${ }^{27}(5 \mu \mathrm{g} / \mathrm{mL})$ (Invitrogen), were dissolved in ice-cold medium (DMEM w/o phenol red) and added to the apical side of bEnd.3 cell monolayers together with the TAMRA-claudin5 peptide $(1-200 \mu \mathrm{M})$. Monolayers were first kept at $4{ }^{\circ} \mathrm{C}$ for $30 \mathrm{~min}$ to allow tracer accumulation on the cell surface and then incubated for 0-30 min at $37^{\circ} \mathrm{C}$. All samples were viewed on a confocal laser scanning microscope.

\section{Immunofluorescence}

The following antibodies were used: anti-Rab34 (Abcam, Boston, MA), -caveolin- 1 , and - $\alpha$-adaptin antibodies (BD Biosciences, Franklin Lakes, NJ) and secondary antibodies antimouse-FITC and -rabbitFITC (Vector laboratories, Burlingame, CA) ${ }^{28}$ Samples were fixed in $4 \%$ paraformaldehyde and then preincubated in the blocking solution containing $5 \%$ normal goat serum, $5 \%$ bovine serum albumine, and $0.05 \%$ Tween 20 in phosphate-buffered saline. Samples were then incubated with primary antibodies overnight at $4{ }^{\circ} \mathrm{C}$. Reactions were visualized by fluorescence-conjugated antimouse and/or antirabbit antibodies. All samples were viewed on a confocal laser scanning microscope.

\section{Evaluation of the endocytosis rate}

To quantify the endocytosis rate of the peptide with or without inhibitors, cells from four independent experiments were analyzed. For visualization of TAMRA-labeled peptides, excitation was performed at $543 \mathrm{~nm}$. All wavelengths greater than $560 \mathrm{~nm}$ were collected as emission signal. For each treatment at least 15 pictures with an average number of $24.1 \pm 0.4$ cells were taken and the fluorescence intensity of the internalized TAMRA-labeled peptide was determined by Zeiss LSM Image Browser Software (Zeiss, Germany). For quantitation of the endocytosis, the emission signals of TAMRA were used. The analysis of each picture provided by the LSM software resulted in a histogram of the emission intensities (0-255 relative fluorescence units, $\mathrm{rfu})$, in which the background ( $<150 \mathrm{rfu})$ and saturated signals $(>254$ $\mathrm{rfu}$ ) were excluded. All signal events of a histogram were added up to the number of signal events of a picture. To allow comparison between different pictures the events were normalized to the number of the cells analyzed.

\section{Evaluation of colocalization}

To quantify the colocalization of claudin- 5 peptide and tracers or vesicle markers, each z-optical section was analyzed using the colocalization finder plug-in of ImageJ (National Institutes of Health, Bethesda, MD). Cells from three independent experiments and three areas per experiment were analyzed. The background contribution to colocalization was corrected using the formula: corrected colocalization $=$ measured colocalization - background colocalization/1 - background colocalization/100. The colocalization probability was estimated by Pearson's correlation coefficient $\left(R_{r}\right)$. The Pearson coefficient ranges between -1 (perfect negative correlation between two images) to +1 (perfect positive correlation between two images). A coefficient of 0 means no correlation between two images.

\section{Statistical analysis}

Results are shown as mean \pm SEM as well as mean \pm SD obtained by the Mann-Whitney test (two-tailed) and Bonferroni test (two-way ANOVA). Differences were considered as significant if $P<0.05$. 

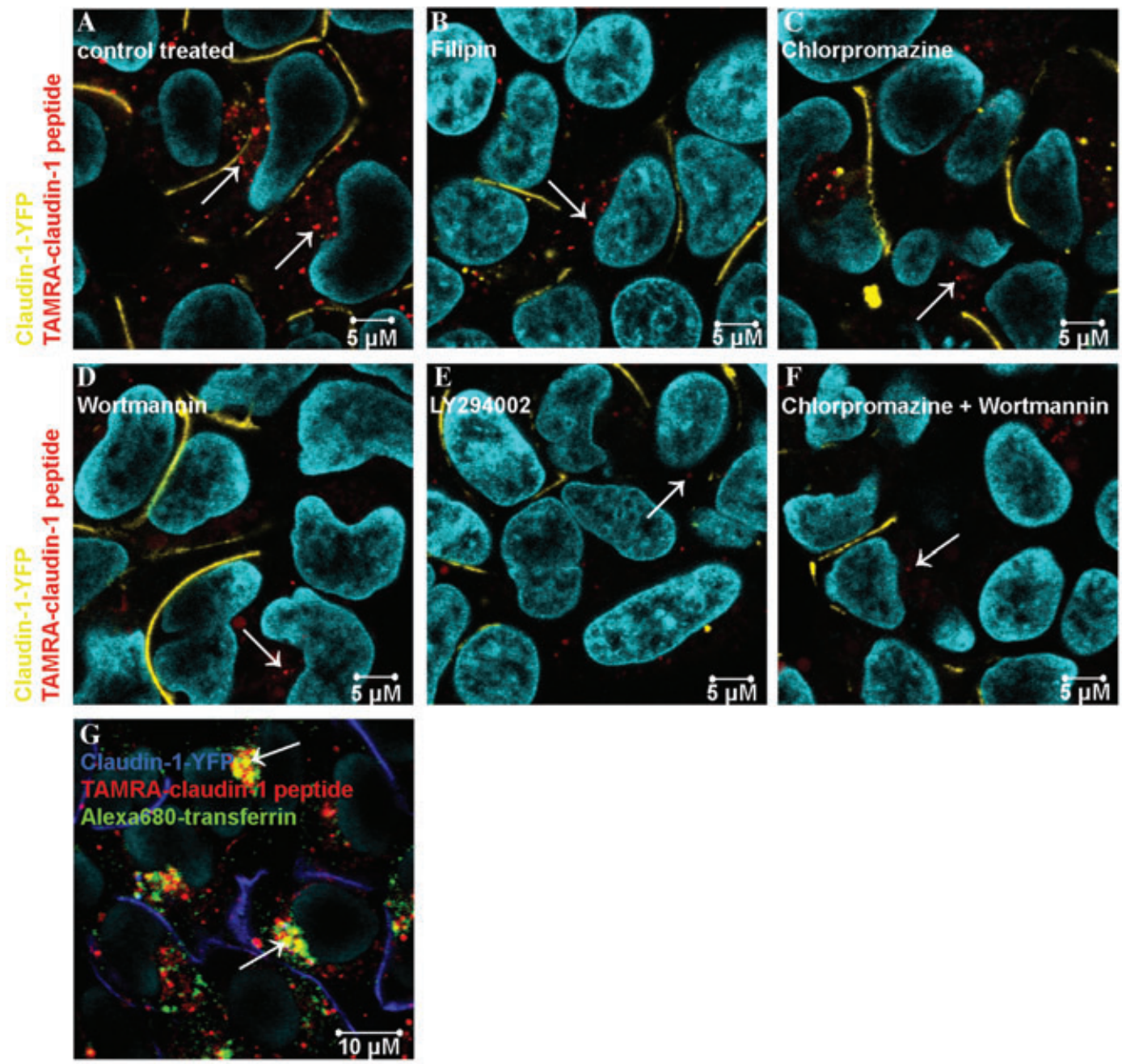

Figure 1. The internalization of the claudin-1 peptide follows the clathrin-mediated and caveolae-mediated endocytosis as well as macropinocytosis. (A) Epithelial human embryonic kidney (HEK)-293 claudin-1 yellow fluorescent protein (YFP) cells were treated with the 5,6-carboxytetramethylrhodamine (TAMRA)-labeled claudin-1 peptide ( $40 \mu \mathrm{M})$ for 35 minutes. The picture shows a clear intracellular distribution of the claudin-1 peptide (red) (arrows). (B-F) HEK-293 claudin-1-YFP cells were pretreated with the corresponding endocytosis inhibitor or inhibitor cocktail. Then, cells were incubated with the claudin-1 peptide (40 $\mu \mathrm{M})$ and the inhibitor or inhibitor cocktail for 35 minutes. Arrows are indicating intracellular localization of the claudin-1 peptide. (B) Filipin, an inhibitor of the caveolae-mediated endocytosis shows a reduction of the cellular uptake of the claudin-1 peptide. (C) Chlorpromazine, an inhibitor of clathrin-mediated endocytosis leads to a higher decrease of intracellular claudin-1 peptide. (D) An inhibition of internalized claudin-1 peptide could be obtained for wortmannin, an inhibitor of macropinocytosis. (E) LY294002, another inhibitor of macropinocytosis, decreases the uptake of the claudin-1 peptide in a lesser amount as compared to wortmannin. (F) The cocktail of the inhibitors chlorpromazine and wortmannin leads to the highest reduction in the cellular uptake of the claudin-1 peptide. (G) Epithelial HEK-293 claudin-1-YFP cells were cotreated with the claudin-1 peptide (red) and Alexa680-tranferrin (green) to determine colocalization. There is a clear colocalization (yellow, arrows) with Alexa680-transferrin, a tracer for the clathrin-mediated endocytosis and the claudin-1 peptide. Nuclei were stained by Hoechst33342 (cyan).

\section{Results}

We synthesized two peptides, TAMRA-claudin-1 and TAMRA-claudin-5, derived from the ECL of the corresponding claudin proteins. The fluorescence dye TAMRA has been introduced N-terminally. The internalization of the claudin-1-derived peptide has been investigated on epithelial HEK-293 cells stably transfected with claudin-1-YFP, whereas the 


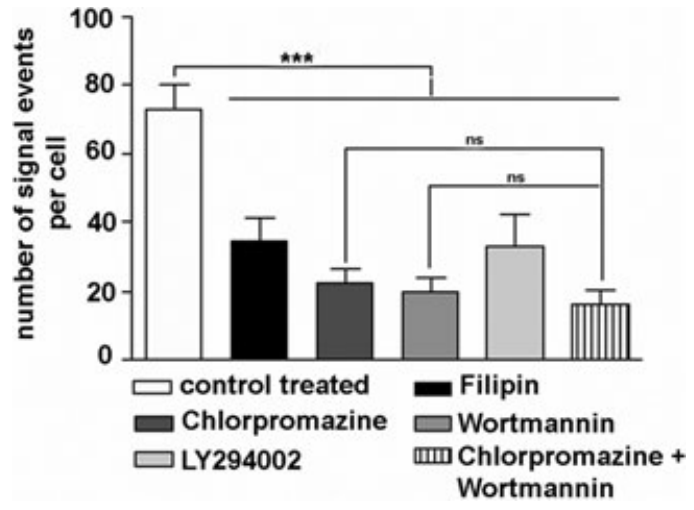

Figure 2. The endocytosis of the claudin-1 peptide decreases by specific pathway inhibitors. For quantification, the number of signal events of the claudin-1 peptide per cell was determined $35 \mathrm{~min}$ after addition of the peptide. All wavelengths greater then $560 \mathrm{~nm}$ were collected as emission signal. For the evaluation, all signal event intensities between 150 and 254 relative fluorescence units were calculated as number of signal events per cell by using the Zeiss LSM Browser Software (Zeiss, Germany). Data are represented as mean \pm SEM; $n=15,{ }^{*} P<$ 0.05 (Mann-Whitney test, one-tailed), ns = not significant.

claudin-5 peptide has been studied on the brain endothelial cell line bEnd.3, endogenously expressing claudin-5. ${ }^{23}$ The HEK cells were used as they exhibit membrane localization of claudin-1 enriched in cell-cell contacts and as the claudin-1 peptide shows a relationship to its full-length protein. The endothelial cells were selected as they express claudin-5 in the cell contacts and as the claudin-5 peptide is related to its mother protein. To study the internalization, we used pharmacological pathway inhibitors to block or decrease the internalization of peptides or applied specific tracers and vesicle markers to determine colocalization of them and the claudin peptide of interest.

For the TAMRA-claudin-1 peptide, Figure 1 $(A-G)$ and Figure 2 show the internalization of the claudin-1 peptide with and without pathway inhibitors into transfected HEK-293 cells, the colocalization of the claudin-1 peptide and of a specific tracer as well as the calculated number of signal events per cell. A clear peptide uptake could be observed after incubation of the cells with $40 \mu \mathrm{M}$ TAMRA-claudin-1 peptide for 35 minutes (Fig. 1A, $70-80$ events/cell). The uptake of the peptide by the HEK claudin- 1 cells was time-dependent. After two hours, $\sim 200$ and after five hours, $\sim 250$ events/cell were registered. Saturation was estimated between 300 and 350 events/cell after 6-24 hours.

To find out the uptake mechanism, different pathway inhibitors were applied and the intracellular amount of claudin-1 peptide was quantified by the determination of signal events per cell (for details see the Methods section) (Fig. 2). The lowest difference in the intracellular amount of the claudin-1 peptide compared to nontreated cells was observed for filipin, an inhibitor of the caveolae-mediated endocytosis (Fig. 1B) an uptake pathway expressed in the HEK cells used. ${ }^{33}$ Chlorpromazine, an inhibitor of the clathrin-mediated pathway, significantly decreased the amount of internalized claudin-1 peptide, much higher than filipin (Fig. 1C). Nearly the same amount of internalized claudin-1 peptide was obtained by the inhibition of macropinocytosis using wortmannin (Fig. 1D) as nonreversible inhibitor. Otherwise, the reversible macropinocytosis inhibitor LY294002 showed a less efficacy than wortmannin (Fig. 1E). However, there was still internalized claudin-1 peptide by using pathway inhibitors. The additive effect of chlorpromazine and wortmannin revealed in the highest reduction of intracellularly located claudin-1 peptide (Fig. 1F). To confirm that the clathrin-mediated endocytosis is involved in the peptide internalization, cells were coincubated with the claudin-1 peptide and transferrin a tracer for this endocytosis pathway. A colocalization of the peptide and the tracer was observed (Fig. 1G) with a correlation coefficient between 0.4 and 0.5. However, not all red fluorescent peptide internalized showed overlay with the internalized green fluorescent transferrin due to the other uptake mechanisms found, such as macropinocytosis and the caveolae pathway.

For the TAMRA-claudin-5 peptide, Figure 3 $(A-D)$ and Figure $4(A-D)$ show the internalization and colocalization of the claudin-5 peptide in bEnd. 3 cells and tracers as well as vesicle markers and the calculated Pearson's correlation coefficients. An adequate peptide uptake could be observed for incubation with $40 \mu \mathrm{M}$ of TAMRAclaudin-5 for 10-30 minutes. To determine the endocytosis pathway several tracers have been used and the colocalization of the corresponding tracer and the claudin-5 peptide has been investigated. No colocalization was determined for transferrin, a tracer for clathrin-mediated endocytosis (Fig. $3 \mathrm{~A})$. On the other hand, the claudin-5 peptide 


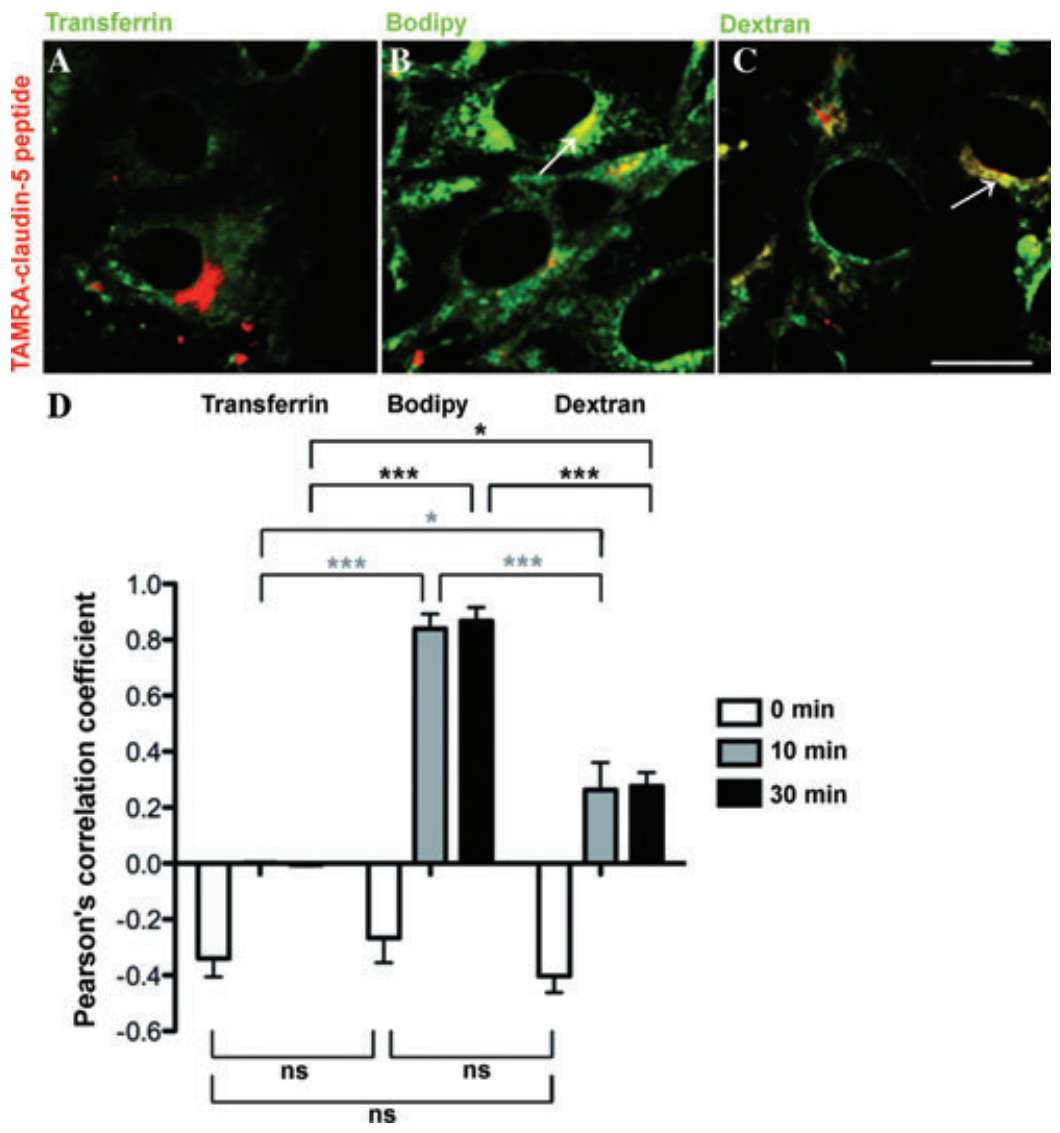

Figure 3. The claudin-5 peptide is colocalized with tracers for the caveolae-mediated endocytosis and macropinocytosis. (A-D) Immortalized mouse brain endothelial cells (bEnd.3) were cotreated with the 5,6-carboxytetramethylrhodamine (TAMRA)-labeled claudin-5 peptide (red) and the corresponding tracer (green) to determine colocalization. (A) bEnd.3 cells were treated with the claudin-5 peptide $(40 \mu \mathrm{M})$ for 10 and 30 minutes. The picture shows no colocalization of the claudin-5 peptide and transferrin, a tracer for clathrin-mediated endocytosis. (B) Bodipy, a tracer for caveolae-mediated endocytosis is clearly colocalized with the claudin-5 peptide (arrow). (C) A less efficient colocalization is given for dextran, a tracer of macropinocytosis (arrow). (D) Evaluation of the Pearson's correlation coefficients of the tracers and the claudin- 5 peptide. Data are represented as mean \pm SD, $n=9 ;{ }^{*},{ }^{* *},{ }^{* * *}, P<0.05,0.01$, and 0.001 , respectively; ns, not significant, $P>0.05$ (Bonferroni test, two-way ANOVA). Scale bar, $20 \mu \mathrm{m}$.

clearly colocalized with the tracer bodipy, which is internalized via caveolae-mediated endocytosis (Fig. 3B). In a smaller amount, a colocalization of the macropinocytosis tracer dextran and the claudin-5 peptide was observed (Fig. 3C). The less the concentration of peptide used the less was the amount of macropinocytosis as endocytosis pathway (data not shown). To have a closer look to the intracellular compartment of internalized claudin-5 peptide, different specific vesicle markers have been used. The $\alpha$-adaptin marker for the clathrin-mediated endocytosis revealed no colocalization with the claudin-5 peptide (Fig. 4B). In contrast, there was a significant correlation between the caveolin-1-GFP and Rab34 marker with the claudin-5 peptide, which confirmed the endocytosis pathways determined by the tracer studies (Fig. 4A and C). In separate experiments, HEK-293 cells transfected with claudin-5-YFP showed an internalization of the claudin- 5 peptide, whereas, the uptake by the nontransfected cells was negligible. This is in agreement with the imagination that the peptide internalization is related to that of the claudin protein. 


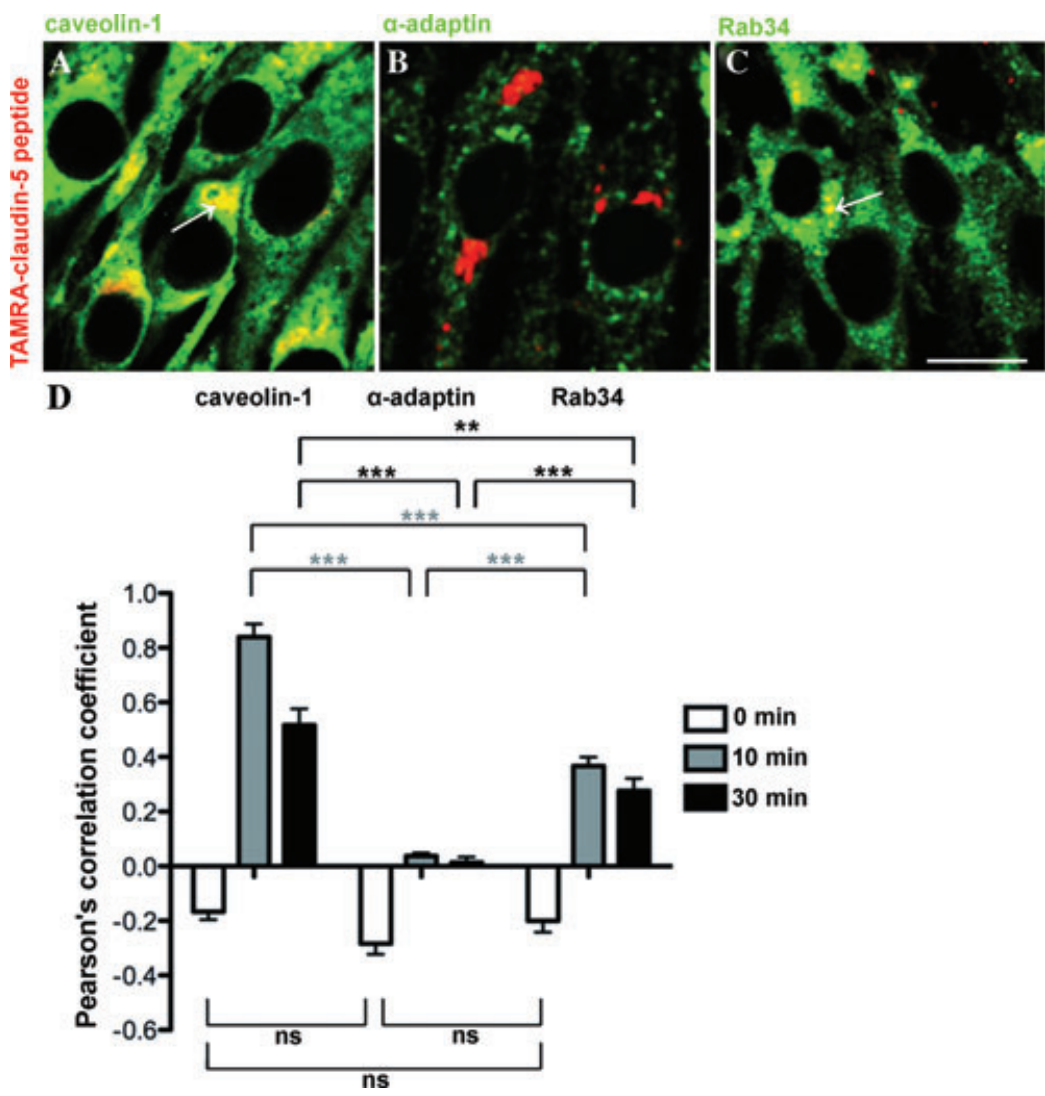

Figure 4. The claudin-5 peptide is colocalized with vesicle markers for the caveolae-mediated endocytosis and macropinocytosis. (A-D) Immortalized mouse brain endothelial cells (bEnd.3) were cotreated with the 5,6-carboxytetramethylrhodamine (TAMRA)labeled claudin-5 peptide (red) and the corresponding vesicle marker (green) to determine colocalization. (A) The vesicle marker caveolin-1 is colocalized with the claudin-5 peptide (arrow). (B) No colocalization is shown for $\alpha$-adaptin, a tracer for the clathrinmediated endocytosis and the peptide. (C) A less efficient colocalization is given for the macropinocytosis vesicle marker Rab34 and the claudin-5 peptide (arrow). (D) Evaluation of the Pearson's correlation coefficients of the vesicle markers and the claudin-5 peptide. Data are represented as mean $\pm \mathrm{SD}, n=9$; ${ }^{* *},{ }^{* * *} P<0.01$ and 0.001 , respectively; ns, not significant, $P>0.05$ (Bonferroni test, two-way ANOVA). Scale bar, $20 \mu \mathrm{m}$.

\section{Discussion}

Here we demonstrate that a claudin-1-derived peptide and a claudin-5-derived peptide are internalized within the first $35 \mathrm{~min}$ into epithelial and endothelial cells expressing the corresponding claudin-subtype; that the claudin-1-derived peptide is internalized by the clathrin-mediated endocytosis and macropinocytosis as well as to a lower amount by the caveolae-mediated pathway; and that the claudin-5-derived peptide is taken up by caveolae-mediated endocytosis and in a lesser amount by macropinocytosis, but not by the clathrin-mediated pathway. These results are predominantly in agreement with earlier studies that showed the same pathways of the respective full-length claudin proteins after extracellular stimuli. ${ }^{4-11}$ Only the partly caveolae-mediated pathway for claudin-1 has not been described in earlier studies. Otherwise, here we present the internalization of claudin peptides derived from the respective ECL instead of the full-length claudin proteins and could determine an internalization without any extracellular stimulus. The finding that the peptides use the same pathways as their full-length proteins supports the assumption that the peptides are internalized together with their proteins. This conclusion is in agreement with the reports that the ECL, where the peptides are derived from, mediates the cell-to-cell association. ${ }^{21,29}$ Nevertheless, it cannot be excluded that the uptake of the peptide segments may also 
occur independently from the uptake of the whole protein.

The internalization and redistribution of TJ proteins is an important function for the migration of epithelial and endothelial cells as well as vessels and tubuli formed by epithelia and endothelia. Furthermore, for selective paracellular barrier opening, which can enhance and/or allow drug delivery, it is necessary to understand the exact mechanism of TJ protein endocytosis. Moreover, to focus on the internalization behavior of claudin proteins, claudin-1, which can be overexpressed in colon cancer cells ${ }^{30}$ and claudin-5, which is a major TJ protein of the blood-brain barrier are promising claudin targets. ${ }^{17,28}$ Thus, claudin-1 peptides conjugated with cytostatic agents could be a suitable approach to be tested as antitumor agent. Claudin-5 peptides could have the potential to interfere with the blood-brain barrier to facilitate drug delivery.

Recently, it has been described that the internalization of TJ proteins is not only depending on an extracellular stimulus given, but also on the cell line and the TJ protein. ${ }^{4}$ In this study, peptides of two different claudin subtypes are internalized in different pathways which confirm the relevance of claudin-subtype specificity in terms of the internalization. In addition, it can be assumed that after inhibition of a specific endocytosis pathway, substances (for example, peptides or proteins) can be internalized via another mechanism. This could explain our results of the claudin-1 peptide by using different inhibitors which shows still internalized claudin-1 peptide even if an endocytosis pathway is blocked. Otherwise, a cross-talk between different endocytosis pathways can also not be excluded. ${ }^{4}$ Here, both claudin peptides are internalized by, at least, two endocytosis pathways. In recent findings of the claudin-5 internalization via caveolae-mediated endocytosis, a noncaveolae-typical early endosome antigen 1 vesicle localization has been observed. ${ }^{4}$ The macropinocytosis is both a nonselective and an actin-dependent internalization mechanism. ${ }^{7}$ The uptake of large macromolecules is often described via this endocytosis pathway in which it can take place by an extracellular stimulation or via a constitutive process. ${ }^{31}$ This could explain the observation of this mechanism for both peptides, the claudin1 and the claudin- 5 peptide, which has also been described for several TJ proteins in the earlier lit- erature. ${ }^{4,10,12}$ The subcellular localization can take place in early endosomes, recycling endosomes, or lysosomes. ${ }^{32}$ It has been found that, in most cases, TJ proteins are first located into early endosomes, followed by a recycling and a transport back to the cell membrane. ${ }^{4}$ It seems to be that the redistribution of proteins plays a more important role than the degradation or de novo synthesis of them. ${ }^{4}$

In summary, we show that a claudin-1 and a claudin-5 peptide, derived from the ECL of their corresponding claudin subtypes, are rapidly internalized by distinct endocytosis pathways. The claudin-1 peptide is internalized by the clathrinmediated endocytosis and macropinocytosis as well as to a lower amount by the caveolae-mediated endocytosis. The uptake of the claudin-5 peptide is caveolae-mediated, whereas macropinocytosis seems to be a less effective uptake mechanism.

\section{Acknowledgments}

We thank Dr. Michael Beyermann for peptide synthesis. This work was supported by the EU project JUSTBRAIN, Else Kröner-FreseniusStiftung 2010'A 52, and DFG BL 408/7-4.

\section{Conflicts of interest}

The authors declare no conflicts of interest.

\section{References}

1. Morita, K. et al. 1999. Claudin multigene family encoding four-transmembrane domain protein components of tight junction strands. Proc. Natl. Acad. Sci. USA 96: 511516.

2. Furuse, M. et al. 1993. Occludin: a novel integral membrane protein localizing at tight junctions. J. Cell Biol. 123: $1777-$ 1788.

3. Wu, J. et al. 2006. Identification of new claudin family members by a novel PSI-BLAST based approach with enhanced specificity. Proteins 65: 808-815.

4. Stamatovic, S.M. et al. 2009. Caveolae-mediated internalization of occludin and claudin-5 during CCL2-induced tight junction remodeling in brain endothelial cells. J. Biol. Chem. 284: 19053-19066.

5. Shen, L. \& J.R. Turner. 2005. Actin depolymerization disrupts tight junctions via caveolae-mediated endocytosis. Mol. Biol. Cell 16: 3919-3936.

6. Marsh, M. \& H.T. McMahon. 1999. The structural era of endocytosis. Science 285: 215-220.

7. Amyere, M. et al. 2002. Origin, originality, functions, subversions and molecular signalling of macropinocytosis. Int. J. Med. Microbiol. 291: 487-494.

8. Sandvig, K. et al. 2011. Clathrin-independent endocytosis: mechanisms and function. Curr. Opin. Cell Biol. 23: 413420. 
9. Capaldo, C.T. \& A. Nusrat. 2009. Cytokine regulation of tight junctions. Biochim. Biophys. Acta. 1788: 864-871.

10. Bruewer, M. et al. 2005. Interferon-gamma induces internalization of epithelial tight junction proteins via a macropinocytosis-like process. Faseb J. 19: 923-933.

11. Ivanov, A.I., A. Nusrat \& C.A. Parkos. 2004. The epithelium in inflammatory bowel disease: potential role of endocytosis of junctional proteins in barrier disruption. Novartis Found Symp. 263: 115-124; Discussion 124-132, 211-218.

12. Ivanov, A.I., A. Nusrat \& C.A. Parkos. 2004. Endocytosis of epithelial apical junctional proteins by a clathrin-mediated pathway into a unique storage compartment. Mol. Biol Cell. 15: 176-188.

13. Hopkins, A.M. et al. 2003. Constitutive activation of Rho proteins by CNF-1 influences tight junction structure and epithelial barrier function. J. Cell Sci. 116: 725-742.

14. Mineta, K. et al. 2011. Predicted expansion of the claudin multigene family. FEBS Lett. 585: 606-612.

15. Furuse, M. et al. 1998. A single gene product, claudin-1 or -2 , reconstitutes tight junction strands and recruits occludin in fibroblasts. J. Cell Biol. 143: 391-401.

16. Colegio, O.R. et al. 2003. Claudin extracellular domains determine paracellular charge selectivity and resistance but not tight junction fibril architecture. Am. J. Physiol. Cell Physiol. 284: C1346-C1354.

17. Nitta, T. et al. 2003. Size-selective loosening of the bloodbrain barrier in claudin-5-deficient mice. J. Cell Biol. 161: 653-660.

18. Furuse, M. et al. 2002. Claudin-based tight junctions are crucial for the mammalian epidermal barrier: a lesson from claudin-1-deficient mice. J. Cell Biol. 156: 1099-1111.

19. Krause, G. et al. 2008. Structure and function of claudins. Biochim. Biophys. Acta. 1778: 631-645.

20. Piontek, J. et al. 2011. Elucidating the principles of the molecular organization of heteropolymeric tight junction strands. Cell Mol. Life Sci. 68: 3903-3918.

21. Piontek, J. et al. 2008. Formation of tight junction: deter- minants of homophilic interaction between classic claudins. FASEB J. 22: 146-158.

22. Graham, F.L. et al. 1977. Characteristics of a human cell line transformed by DNA from human adenovirus type 5. J. Gen. Virol. 36: 59-74.

23. Omidi, Y. et al. 2003. Evaluation of the immortalised mouse brain capillary endothelial cell line, b.End3, as an in vitro blood-brain barrier model for drug uptake and transport studies. Brain Res 990: 95-112.

24. Wiranowska, M., L.O. Colina \& J.O. Johnson. Clathrinmediated entry and cellular localization of chlorotoxin in human glioma. Cancer Cell Int. 11: 27.

25. Magzoub, M. et al. 2006. N-terminal peptides from unprocessed prion proteins enter cells by macropinocytosis. Biochem. Biophys. Res. Commun. 348: 379-385.

26. Sabah, J.R. et al. 2007. Transcytotic passage of albumin through lens epithelial cells. Invest. Ophthalmol. Vis. Sci. 48: 1237-1244.

27. Gottlieb, T.A. et al. 1993. Actin microfilaments play a critical role in endocytosis at the apical but not the basolateral surface of polarized epithelial cells. J. Cell Biol. 120: 695-710.

28. Stamatovic, S.M., R.F. Keep \& A.V. Andjelkovic. Tracing the endocytosis of claudin-5 in brain endothelial cells. Methods Mol. Biol. 762: 303-320.

29. Daugherty, B.L. et al. 2007. Regulation of heterotypic claudin compatibility. J. Biol. Chem. 282: 30005-30013.

30. Singh, A.B. et al. 2011. Claudin-1 up-regulates the repressor ZEB-1 to inhibit E-cadherin expression in colon cancer cells. Gastroenterology 141: 2140-2153.

31. Kerr, M.C. \& R.D. Teasdale. 2009. Defining macropinocytosis. Traffic 10: 364-371.

32. Kirkham, M. \& R.G. Parton. 2005. Clathrin-independent endocytosis: new insights into caveolae and non-caveolar lipid raft carriers. Biochim. Biophys. Acta. 1745: 273-286.

33. Cha, S.-K. et al. 2011. Calcium-sensing receptor decreases cell surface expression of the inwardly rectifying $\mathrm{K}^{+}$channel Kir4.1. J. Biol. Chem. 286: 1828-1835. 